\title{
Relationship between cigarette smoking and nutrient intakes and blood status indices of older people living in the UK: further analysis of data from the National Diet and Nutrition Survey of people aged 65 years and over, 1994/95
}

\author{
CM Walmsley, CJ Bates*, A Prentice and TJ Cole \\ MRC Human Nutrition Research, Downhams Lane, Milton Road, Cambridge CB4 1XJ, UK
}

Submitted 5 June 1998: Accepted 28 July 1998

\begin{abstract}
Objectives: To examine the relationship between cigarette smoking and a range of nutrient intakes and blood status indices in older people.

Design: National Diet and Nutrition Survey: cross-sectional survey of nationally representative sample of people aged 65 years and over.

Setting: Mainland Britain during 1994/95.

Subjects: 1191 people (619 male, 572 female) aged 65 years and over, of whom 920 were living in private households and 271 were living in institutions.

Results: Cigarette smoking was inversely correlated with intakes of antioxidants and other micronutrients after adjustment for age, sex and domicile. Cigarette smoking was also inversely correlated with a number of antioxidant micronutrient status indices including plasma vitamin $\mathrm{C}$ and the carotenoids (but not vitamin $\mathrm{E}$ status indices), and with other micronutrient status indices, including plasma pyridoxal phosphate, red cell and serum folate, after adjustment for age, sex, domicile and the corresponding nutrient intake. Previous cigarette smoking or cigar/pipe smoking was not generally associated with lower nutrient intakes or status indices, however, both current and previous cigarette smoking was associated with increased concentrations of acute phase indicators. Further adjustment for total energy intake and/or sociodemographic, health and drug usage variables attenuated only a few of the associations observed.

Conclusion: Older people who smoke cigarettes are at increased risk of suboptimal antioxidant and other micronutrient intakes and status, but the lower intakes found in cigarette smokers only partly explain their reduced blood indices.
\end{abstract}

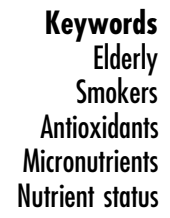

Many studies of cigarette smokers have reported lower dietary intakes ${ }^{1-3}$ and lower blood concentrations of certain antioxidants ${ }^{4-8}$ and other micronutrients such as folate ${ }^{9-11}$, and this may contribute to the increased risk of vascular disease ${ }^{12}$ and certain cancers ${ }^{13}$ observed in smokers. Cessation of cigarette smoking resulted in acute improvements in plasma antioxidant status ${ }^{14}$ and progressively improved nutrient intakes with time since cessation of smoking ${ }^{15}$.

The abundance of free radicals in, or produced by, cigarette smoke may induce an oxidative stress on both the respiratory and circulatory systems ${ }^{16}$, and the lower blood levels of antioxidants found in smokers may partly be a consequence of a greater antioxidant depletion due to a sustained smoke-related oxidant load $^{12}$.

Mechanisms to explain the adverse effects of smoking on non-antioxidant micronutrients such as folate and vitamin $B_{12}$ are less well studied but some of the chemical agents in cigarette smoke have been shown to inactivate these nutrients ${ }^{13}$; other possible mechanisms may be via destruction or displacement from the circulation ${ }^{10}$.

Recent supplementation trials have been carried out to test the effectiveness of pharmacological doses of specific antioxidants particularly vitamin $\mathrm{E}$ and $\beta$ carotene, and other micronutrients such as folate, on the potential to reduce the incidence of cigarette smoke-related diseases ${ }^{17-22}$, but the results of these studies have so far been inconclusive ${ }^{23}$. It has recently been suggested that daily consumption of a variety of fruits and vegetables, a natural complex source of carotenoids, may remain the better way to help reduce the risk of cancers and cardiovascular diseases in both smokers and non-smokers ${ }^{24}$.

Little of the research into the adverse effects of 
smoking has been carried out in older people. This population group are a vulnerable group with respect to micronutrient deficiencies for various reasons including decreasing food intakes ${ }^{25}$ and impaired metabolism ${ }^{26}$, higher incidence of disease and drug administration. A decrease in blood levels of vitamin C, $\beta$-carotene ${ }^{27}$ and folate ${ }^{9}$ with old age was reported to be greater in smokers compared to non-smokers, independent of nutrient intake.

A cross-sectional National Diet and Nutrition Survey of people aged 65 years and over was carried out in mainland Britain during 1994-95, and included assessment of food and nutrient intakes and a wide range of nutrient and other blood status indices. This provided the opportunity to examine the relationships between smoking and a range of antioxidant and other nutrient intakes and status indices in a large sample of older people and to consider the results in terms of their implications for dietary requirements for micronutrients.

\section{Subjects and methods}

Data from the National Diet and Nutrition Survey $(\mathrm{NDNS})^{28}$ of people aged 65 years or over in mainland Britain were used in this study of the relationships between smoking and nutrient intakes and status of older people. The NDNS was commissioned by the British government (Department of Health and the Ministry of Agriculture, Fisheries and Food) and carried out during the period October 1994 to September 1995. Social and Community Planning Research (SCPR) selected a sample of older people, from 80 postcode sectors within Britain, using a stratified random design. Both those living in private households ( $79 \%$ of total) and in institutions, i.e. residential and nursing homes ( $21 \%$ of total) were represented in each sector. For statistical adequacy, predetermined numbers were recruited from both sexes and from each of the following age groups: $65-74$ years, $75-84$ years and $85+$ years, and this required over-sampling of male subjects and of the older age groups. Seventy-eight per cent $(n=2060)$ of the eligible sample, selected to be included in the survey, took part. Of these, $82 \%$ provided a 4 -day dietary record and $60 \%$ provided a blood sample for nutrients analysed in this study.

Information on lifestyle, health, and eating and drinking habits of the participants was obtained through a structured interview carried out by trained fieldworkers. As part of this interview participants were asked if they had ever smoked cigarettes, a cigar or a pipe; if they currently smoked, and if so, how many cigarettes/cigars they smoked daily; and for those who had given up cigarettes, how long ago they had stopped smoking. Following the interview those living in private households were asked to keep a weighed record of all food and drink (including alcohol) consumed over 4 consecutive days. For those subjects unable or unwilling to keep a weighed record, a descriptive diary, with household measures and portion sizes, was kept by the participant or carer, and weights were assigned by the survey nutritionists. Those living in institutions were visited daily for 4 days by the interviewer who weighed one main meal on each visit. The participants, or their carers, were asked to keep a descriptive record of all food and drink consumed for the remainder of each 24-h period. A feasibility study in this age group had previously shown that there were no significant differences in average intakes of key nutrients between a 7- or a 4-day dietary record, but levels of compliance and completion of the record were higher in those keeping the 4 -day record ${ }^{29}$. The feasibility study showed intakes of $\alpha$ - and $\beta$ carotene and total fat intake varied according to the day of the week. It was considered essential to have records for all days of the week in the main survey and thus a dietary record placement pattern was selected which would ensure an even spread of start days throughout the week ${ }^{28}$.

A trained nurse obtained the following in the participants home: anthropometric and grip strength measurements, information on the use of medications, blood pressure measurement and an early morning blood sample, usually fasting. The blood sample was taken after the 4-day diet record generally within a few days, and no more than 2 weeks later. Analyses for haematology and haematinic nutrients (including serum folate and vitamin $\mathrm{B}_{12}$ ) were performed at Addenbrooke's Haematology Laboratory, Cambridge, UK, and other biochemical index measurements were performed at the MRC Human Nutrition Research, Cambridge, UK. The vitamin status index and other biochemical assay procedures are reported elsewhere ${ }^{28}$.

The analysis reported here was restricted to those 1191 participants who provided a full 4-day dietary diary, a blood sample and information on smoking. Preliminary analysis showed that those who did not provide this information $(n=869)$ differed demographically (i.e. they were more likely to be $85+$ years than $<85$ years, $P<0.01$; female than male, $P<0.0001$; and live in Scotland and northern England than central and south-west England, $P<0.05$ ), but the provision of a blood sample or a 4-day diary was not significantly associated with smoking status. A weighting variable was applied to mean values, to correct in the private household sample for disproportionate sampling of sex and age groups, over-representation of people living alone and different co-operation rates between subgroups for region, sex and age. The institution sample was weighted to correct for disproportionate sampling of institutions by area and of individuals within institutions. Thus mean values (but not numbers of 
Table 1 Proportion of study participants by smoking status*

\begin{tabular}{|c|c|c|c|c|c|c|}
\hline & \multicolumn{2}{|c|}{ Males } & \multicolumn{2}{|c|}{ Females } & \multicolumn{2}{|c|}{ All participants } \\
\hline & $n$ & $\%$ & $n$ & $\%$ & $n$ & $\%$ \\
\hline \multicolumn{7}{|l|}{ Non-smokers } \\
\hline Never smoked cigarettes & 94 & 15 & 261 & 46 & 355 & 30 \\
\hline Previously smoked cigarettes & 380 & 61 & 242 & 42 & 622 & 52 \\
\hline $\begin{array}{l}\text { Current smokers } \\
<20 \text { cigarettes day }^{-1 \dagger}\end{array}$ & 71 & 11 & 57 & 10 & 128 & 11 \\
\hline$\geqslant 20$ cigarettes day $^{-1 \dagger}$ & 35 & 6 & 12 & 2 & 47 & 4 \\
\hline Cigar/pipe smokers & 39 & 6 & - & - & 39 & 3 \\
\hline Total & 619 & 100 & 572 & 100 & 1191 & 100 \\
\hline
\end{tabular}

* Excludes those without a full 4-day diet diary and those without a blood sample.

${ }^{\dagger}$ Average number of cigarettes smoked: $<20$ day $^{-1}=9 ; \geqslant 20$ day $^{-1}=22$; all cigarette smokers $=12$.

Smokers using both cigarettes and cigars/pipe are classed as cigarette smokers.

subjects) are based on a representative sample of people aged 65 years and over in mainland Britain. Participants who reported taking supplements of the nutrient in question during the 4-day record were excluded from the analysis to prevent severe skewness of the data. Preliminary analysis for key nutrients, such as vitamins $\mathrm{C}, \mathrm{E}$ and $\mathrm{D}$ and folate, showed that the proportion of supplement takers did not differ significantly between cigarette smokers and non-cigarette smokers $(P>0.1)$. Data reduction was performed using Microsoft Excel and data analysis was carried out using DataDesk computer programs (Data Description, Inc.). General linear models were used to perform linear tests for trends (from analysis of variance), and multiple regression analysis for which $P \leqslant 0.01$ was deemed to be statistically significant. Logistic regression and analysis of variance were used to test differences between means using the Scheffe test for which $P \leqslant 0.05$ was considered significant. Estimate of the extra vitamin $\mathrm{C}$ intake required by cigarette smokers was determined by the ratio of the smoking coefficient divided by an interaction coefficient (smoking by dietary intake of vitamin C), with the standard error calculated by Fieller's theorem. Variables were transformed to normality where necessary, using either logarithmic, square root or reciprocal transformations. Means are presented after adjustment for the effects of age, sex and domicile (whether living in private households or institutions), and nutrient status indices are also adjusted for nutrient intake. Results of further adjustment for total energy intake and/or sociodemographic (social class, region, receipt of benefits), health (self-reported health, depression score) and drug usage (cardiovascular, gastrointestinal, respiratory and infection) variables are reported in the text.

\section{Results}

Table 1 shows that of the 1191 participants in this study $15 \%$ currently smoked cigarettes, the majority of whom were light smokers ( $<20$ cigarettes/day). About half of all participants, and more males than females, were previous cigarette smokers, and almost one-third, the majority of whom were women, had never smoked cigarettes.

The association between group characteristics and cigarette smoking is shown in Table 2 . The probability of smoking cigarettes was greater in 65-74 year olds than those 75 years and over, and in males than females. After adjustment for age, sex and domicile, results showed cigarette smoking was more likely in those from manual classes than non-manual, those who received benefits (excluding pensions) than those who did not, those living in Scotland and northern England than central and south-west England, those who reported themselves to be in bad health than those in good health, those with less depression, those not taking cardiovascular and gastrointestinal drugs, and those taking respiratory drugs compared to those not taking them. Of those who smoked cigarettes, heavier smoking was observed in the 65-74 year olds compared to those over 75 years, in males than females, in those living in private households than those in institutions, and in those taking drugs for infections (not presented).

The relationship between smoking status and the probability of consuming different food groups is shown in Table 3, adjusted for age, sex and domicile. Cigarette smoking was more common in those who reported consuming white bread and whole milk during the 4-day dietary record, and less so in those consuming all types of breakfast cereal, semi-skimmed milk, fish and fruit. After further adjustment for sociodemographic and health variables, significance fell outside the $1 \%$ level $(P>0.01)$ for white bread $(P=0.03)$, breakfast cereal other than wholegrain and high fibre $(P=0.03)$ and fish $(P=0.05)$. Previous cigarette and cigar/pipe smokers consumed the different food groups to a similar extent to those who had never smoked cigarettes after adjustment for age, sex and domicile (not presented).

Table 4 shows mean nutrient intakes in nonsmokers and current smokers, after adjustment for 
Table 2 Relationship between group characteristics and cigarette smoking*

\begin{tabular}{|c|c|c|c|c|c|}
\hline \multirow[b]{2}{*}{ Group characteristics } & \multicolumn{3}{|c|}{$\begin{array}{l}\text { Percentage of group } \\
\text { who smoke cigarettes }\end{array}$} & \multirow[b]{2}{*}{ OR $(95 \% \mathrm{Cl})$} & \multirow[b]{2}{*}{$P$ value } \\
\hline & $A \%$ & $\mathrm{~B} \%$ & $\mathrm{C} \%$ & & \\
\hline Age (A 65-74; B 75-84; C 85+ years) & 24 & 14 & 4 & $8.6(4.4,16.7)$ & $<0.0001$ \\
\hline Sex (A male; $B$ female) & 18 & 12 & - & $1.6(1.2,2.3)$ & 0.004 \\
\hline Domicile ( $A$ free-living; $B$ in an institution) & 16 & 14 & - & $1.2(0.8,1.7)$ & 0.5 \\
\hline Social class ${ }^{\ddagger}$ (A non-manual; B manual) & 8 & 14 & - & $1.9(1.3,2.7)$ & 0.001 \\
\hline $\begin{array}{l}\text { Receipt of benefits (excl. pensions })^{\ddagger} \\
\quad(\text { A any received; } B \text { none received) }\end{array}$ & 14 & 8 & - & $1.9(1.3,2.7)$ & 0.0007 \\
\hline $\begin{array}{l}\text { Region }{ }^{\ddagger} \text { (A Scotland \& North England; B central \& SW England; } \\
\text { C London \& SE England) }\end{array}$ & 16 & 9 & 11 & $1.9(1.3,2.9)$ & 0.004 \\
\hline Self-reported health ${ }^{\ddagger}$ (A very good/good; B fair; C bad/very bad) & 10 & 13 & 22 & $2.6(1.3,4.8)$ & 0.02 \\
\hline Depression $^{\ddagger}(A>6$; B $0-6$ negative statements $)$ & 11 & 15 & - & $1.5(1.0,2.2)$ & 0.03 \\
\hline Alcohol consumption ${ }^{\ddagger, \|}$ (A none; B some) & 12 & 11 & - & $1.1(0.8,1.6)$ & $0.5^{\|}$ \\
\hline Physical activity ${ }^{\ddagger}$ (A inactive/light $B$ moderate/vigorous) & 10 & 13 & - & $1.3(0.8,2.1)$ & 0.3 \\
\hline Cardiovascular drugs $\mathrm{s}^{\ddagger \S}$ (A none; B one or more) & 14 & 9 & - & $1.6(1.1,2.3)$ & 0.007 \\
\hline Respiratory drugs $\ddagger$, $\$$ (A none; B one or more) & 11 & 18 & - & $1.8(1.1,2.9)$ & 0.02 \\
\hline Gastrointestinal drugs $^{\ddagger, \S}$ (A none; B one or more) & 14 & 8 & - & $2.0(1.3,3.2)$ & 0.004 \\
\hline
\end{tabular}

* Excludes those without a full 4-day diet diary and those without a blood sample. Weighted percentages are presented to correct for non-proportional sampling (see subjects and methods section).

${ }^{\dagger}$ Results obtained by logistic regression with smoking as the binary dependent variable $(0=$ non-cigarette smoker; $1=$ cigarette smoker $)$ and group characteristic as the independent variable.

$¥$ Adjusted for age category, sex and domicile.

$\S$ All other drugs analysed (categories: antibiotics, infection, endocrine, cytotoxic, nutrition/blood, muscular/skeletal, skin, aspirin, eye, etc.) showed no significant $(P>0.05)$ associations with the likelihood of smoking.

"Alcohol analysed as a continuous, or categoric independent variable also showed no significant $(P>0.05)$ association.

$\mathrm{OR}$, odds ratio of highest versus lowest percentage within groups A-C.

NS, not significant when $P>0.05$ (Scheffe test).

age, sex and domicile. Cigarette smoking was inversely correlated with intakes of vitamin $\mathrm{C}, \beta$-carotene, total carotene, vitamins $\mathrm{E}, \mathrm{B}_{2}$, and $\mathrm{B}_{6}$, folate, vitamin $\mathrm{B}_{1}$, zinc, iron, vitamin $\mathrm{D}$, calcium, copper, total energy, carbohydrate and non-starch polysaccharides. After further adjustment for total energy intake, sociodemographic, health and drug usage variables, significance fell outside the $1 \%$ level $(P>0.01)$ for vitamin $C(P=0.09)$ and vitamin $\mathrm{B}_{6}(P=0.07)$ (not presented).

A large proportion of participants (20\% male, 31\% female) had energy intakes $<1.06$ basal metabolic rate (BMR) suggesting that, except for the very elderly, the

Table 3 Relationship between smoking status and likelihood of consuming foods in different food groups*

\begin{tabular}{|c|c|c|c|c|c|c|}
\hline \multirow[b]{4}{*}{ Food group } & \multicolumn{2}{|c|}{ Non-smoker } & \multicolumn{3}{|c|}{ Current smoker } & \multirow{4}{*}{$\begin{array}{c}\text { Test for linear trend } \\
\qquad \text { value }\end{array}$} \\
\hline & $\begin{array}{c}\text { Never } \\
\text { smoked } \\
\text { cigarettes }\end{array}$ & $\begin{array}{c}\text { Previously } \\
\text { smoked } \\
\text { cigarettes }\end{array}$ & $\begin{array}{l}\text { Cigar/ } \\
\text { pipe }\end{array}$ & $\begin{array}{c}<20 \\
\text { cigarettes } \\
\text { day }^{-1}\end{array}$ & $\begin{array}{c}\geqslant 20 \\
\text { cigarettes } \\
\text { day }^{-1}\end{array}$ & \\
\hline & \multicolumn{5}{|c|}{$\%$ of group who consumed food from this food group } & \\
\hline & $(n=354)$ & $(n=622)$ & $(n=39)$ & $(n=128)$ & $(n=47)$ & \\
\hline Bread, white & 77 & 76 & 90 & 86 & 87 & 0.006 \\
\hline Bread, wholemeal & 40 & 39 & 30 & 31 & 28 & 0.02 \\
\hline Breakfast cereal, wholegrain and high fibre & 55 & 57 & 63 & 28 & 26 & $<0.0001$ \\
\hline Breakfast cereal, other & 39 & 33 & 21 & 28 & 19 & 0.002 \\
\hline Milk, whole & 50 & 55 & 63 & 67 & 61 & 0.002 \\
\hline Milk, semi-skimmed & 53 & 49 & 49 & 37 & 42 & 0.006 \\
\hline Milk, skimmed & 6 & 6 & 2 & 5 & 3 & 0.3 \\
\hline Red meat, poultry and dishes & 94 & 92 & 82 & 92 & 95 & 0.3 \\
\hline Meat products, meat pies and pasties & 85 & 85 & 85 & 88 & 95 & 0.09 \\
\hline Fish, total $^{\mathrm{a}}$ & 78 & 78 & 82 & 66 & 71 & 0.01 \\
\hline 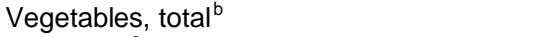 & 98 & 98 & 100 & 94 & 96 & 0.03 \\
\hline Fruit, total $^{\mathrm{c}}$ & 85 & 89 & 80 & 69 & 80 & $<0.0001$ \\
\hline
\end{tabular}

* Adjusted for age category, sex and domicile. Excludes those without a full 4-day diet diary and those without a blood sample. Weighted percentages are presented to correct for non-proportional sampling (see subjects and methods section). Smokers using both cigarettes and cigars/pipe are classed as cigarette smokers.

${ }^{\dagger}$ Across the five smoking groups.

${ }^{a}$ Includes fish dishes; fried and coated fish, shellfish; canned fish.

${ }^{\mathrm{b}}$ Includes raw and cooked potatoes; salad vegetables; vegetable dishes.

${ }^{\mathrm{c}}$ Includes canned fruit.

Not significant when $P>0.01$. 
sick or the physically handicapped, energy intake may have been under-reported ${ }^{30}$. However, the level of under-reporting was similar across the five smoking groups (for males chi-squared $=2.2, \quad P=0.7$; for females chi-squared $=2.9, P=0.09$ ).

Multiple regression analysis suggested that older people who smoke cigarettes need more dietary vitamin $\mathrm{C}$ than non-smokers to obtain equivalent plasma vitamin $\mathrm{C}$ levels. A significant interaction coefficient $(P=0.003)$ suggested that equivalent plasma vitamin $C$ levels of smokers and non-smokers could be obtained at a certain level of vitamin $\mathrm{C}$ intake. The mean extra vitamin $C$ intake needed for plasma levels of cigarette smokers to reach those of nonsmokers was calculated as $112 \mathrm{mg} \mathrm{day}^{-1}(\mathrm{SE}=58)$ but the variance around this mean was wide. Linear regression analysis showed that $35 \%$ of the difference in plasma vitamin $\mathrm{C}$ between cigarette smokers and non-smokers (after adjustment for age, sex and domicile) was attributable to vitamin $\mathrm{C}$ intake, and thus $65 \%$ is attributable to other factors including the metabolic effects of smoking.

Previous cigarette smokers who had given up $\leqslant 2$ years before had lower intakes of vitamin $\mathrm{E}$ than those who had never smoked cigarettes (group A), and those who had given up $>2$ years before (group B), after adjustment for age, sex and domicile. This relationship remained significant (group A $P=0.001$; group B $P=0.003$ ) after further adjustment for total energy intake, sociodemographic, health and drug usage variables (not presented). No other significant differences between previous smokers or cigar/pipe smokers and never smokers with respect to mean nutrient intakes were observed after adjustment for age, sex and domicile.

Table 5 shows mean blood concentrations of a range of antioxidant, other micronutrient and lipid status indices in non-smokers and current smokers, adjusted for age, sex, domicile and corresponding nutrient intake. Cigarette smoking was inversely correlated with plasma vitamin C, $\beta$-carotene, $\alpha$-carotene, lycopene, lutein and $\beta$-cryptoxanthin; directly correlated with erythrocyte glutathione reductase activation coefficient (EGRAC; an increase in this index indicates worsening status of riboflavin); but was not associated with vitamin $\mathrm{E}$ status indices ( $\alpha$-tocopherol and

Table 4 Relationship between smoking status and nutrient intake*

\begin{tabular}{|c|c|c|c|c|c|c|c|}
\hline \multirow[b]{3}{*}{ Nutrient intakes } & \multicolumn{2}{|c|}{ Non-smoker } & \multicolumn{3}{|c|}{ Current smoker } & \multirow{3}{*}{$\begin{array}{l}\text { Test for } \\
\text { linear trend" } \\
P \text { value }\end{array}$} & \multirow[b]{3}{*}{$\begin{array}{l}\text { Residual } \\
\text { SD }^{\text {III }}\end{array}$} \\
\hline & \multirow{2}{*}{$\begin{array}{c}\begin{array}{c}\text { Never } \\
\text { smoked } \\
\text { cigarettes }\end{array} \\
\begin{array}{c}\text { Adj. mean } \\
(n=272-346)\end{array}\end{array}$} & \multirow{2}{*}{$\begin{array}{c}\begin{array}{c}\text { Previously } \\
\text { smoked } \\
\text { cigarettes }\end{array} \\
\begin{array}{c}\text { Adj. mean } \\
(n=477-610)\end{array}\end{array}$} & \multirow{2}{*}{$\begin{array}{c}\begin{array}{c}\text { Cigar/ } \\
\text { pipe }\end{array} \\
\begin{array}{c}\text { Adj. mean } \\
(n=35-39)\end{array}\end{array}$} & \multirow{2}{*}{$\begin{array}{c}<20 \\
\begin{array}{c}<\text { cigarettes } \\
\text { day }^{-1}\end{array} \\
\begin{array}{c}\text { Adj. mean } \\
(n=97-127)\end{array}\end{array}$} & \multirow{2}{*}{$\begin{array}{c}\geqslant 20 \\
\begin{array}{c}\text { cigarettes } \\
\text { day }^{-1}\end{array} \\
\begin{array}{c}\text { Adj. mean } \\
(n=35-47)\end{array}\end{array}$} & & \\
\hline & & & & & & & \\
\hline \multicolumn{8}{|l|}{ Antioxidant micronutrients } \\
\hline Vitamin C $\left(\mathrm{mg} \mathrm{day}^{-1}\right)^{\dagger}$ & 49.0 & 49.2 & 50.2 & 40.1 & 36.7 & 0.0001 & 0.26 \\
\hline$\beta$-carotene $\left(\mu \mathrm{g} \mathrm{day}^{-1}\right)^{\dagger}$ & 1387 & 1321 & 1239 & 989 & 938 & $<0.0001$ & 0.34 \\
\hline Total carotenoids $\left(\mu \mathrm{g} \text { day }^{-1}\right)^{\dagger}$ & 1517 & 1449 & 1352 & 1079 & 1047 & $<0.0001$ & 0.35 \\
\hline Vitamin E $\left(\mathrm{mg} \mathrm{day}^{-1}\right)^{\dagger}$ & 7.04 & 6.90 & 6.53 & 5.91 & 5.02 & $<0.0001$ & 0.24 \\
\hline Vitamin $B_{2}\left(m g \text { day }^{-1}\right)^{\dagger}$ & 1.58 & 1.61 & 1.55 & 1.30 & 1.44 & $<0.0001$ & 0.16 \\
\hline \multicolumn{8}{|l|}{ Other micronutrients } \\
\hline Vitamin $\mathrm{B}_{6}\left(\mathrm{mg} \mathrm{day}^{-1}\right)^{\ddagger}$ & 1.81 & 1.80 & 1.75 & 1.59 & 1.59 & $<0.0001$ & 0.42 \\
\hline Retinol $\left(\mu \mathrm{g}_{\text {day }}{ }^{-1}\right)^{\dagger}$ & 499 & 485 & 455 & 423 & 494 & 0.2 & 0.35 \\
\hline Folate $\left(\mu \mathrm{g} \mathrm{day}^{-1}\right)^{\ddagger}$ & 228 & 228 & 204 & 193 & 194 & $<0.0001$ & 5.25 \\
\hline Vitamin $B_{1}\left(m g \text { day }^{-1}\right)^{\ddagger}$ & 1.33 & 1.29 & 1.24 & 1.12 & 1.00 & $<0.0001$ & 0.33 \\
\hline Vitamin $\mathrm{B}_{12}\left(\mu \mathrm{g} \text { day }^{-1}\right)^{\dagger}$ & 4.32 & 4.29 & 4.60 & 3.80 & 4.07 & 0.07 & 0.25 \\
\hline $\operatorname{Zinc}\left(\mathrm{mg} \operatorname{day}^{-1}\right)^{\ddagger}$ & 7.92 & 7.71 & 7.67 & 6.68 & 7.05 & $<0.0001$ & 0.81 \\
\hline Iron $\left(\mathrm{mg} \mathrm{day}^{-1}\right)^{\dagger}$ & 9.43 & 9.30 & 8.59 & 7.75 & 7.29 & $<0.0001$ & 0.14 \\
\hline Vitamin $\mathrm{D}\left(\mu \mathrm{g} \text { day }^{-1}\right)^{\dagger}$ & 3.12 & 3.15 & 2.94 & 2.35 & 2.09 & $<0.0001$ & 0.28 \\
\hline Calcium (mg day $\left.{ }^{-1}\right)^{\dagger}$ & 836 & 804 & 800 & 664 & 746 & $<0.0001$ & 0.15 \\
\hline Copper $\left(\mathrm{mg} \mathrm{day}^{-1}\right)^{\dagger}$ & 0.883 & 0.869 & 0.819 & 0.731 & 0.788 & $<0.0001$ & 0.17 \\
\hline \multicolumn{8}{|l|}{ Macronutrients and fibre } \\
\hline Total energy (incl. alcohol) $\left(\mathrm{MJ} \mathrm{day}^{-1}\right)$ & 7.48 & 7.36 & 7.67 & 6.91 & 7.10 & 0.004 & 1.68 \\
\hline Fat $\left(\right.$ g day $\left.^{-1}\right)$ & 70.4 & 69.7 & 73.6 & 68.9 & 64.3 & 0.2 & 20.32 \\
\hline Carbohydrate $\left(\mathrm{g}_{\text {day }}{ }^{-1}\right)^{\dagger}$ & 223 & 218 & 217 & 194 & 210 & $<0.0001$ & 0.12 \\
\hline Non-starch polysaccharides $\left(\mathrm{g} \text { day }^{-1}\right)^{\dagger, \S}$ & 16.0 & 15.9 & 14.5 & 12.4 & 12.1 & $<0.0001$ & 0.16 \\
\hline
\end{tabular}

*Adjusted for age category, sex and domicile. Excludes supplement takers, those without a full 4-day diet diary, and those without a blood sample. Weighted means are presented to correct for non-proportional sampling (see subjects and methods section). Analysis of variance used to obtain means with nutrient intakes as the dependent variable and smoking status as the independent variable. Smokers using both cigarettes and cigars/pipe are classed as cigarette smokers. Variables were transformed to obtain a normal distribution, and means back-transformed: ${ }^{\dagger}$ log, ${ }^{\ddagger}$ square root.

$\S$ Englyst method.

"Across the five smoking groups

III Overall residual standard deviation on the transformed scale where relevant.

Adj., adjusted. Not significant when $P>0.01$. 
Table 5 Relationship between smoking status and micronutrient and lipid status indices*

\begin{tabular}{|c|c|c|c|c|c|c|c|}
\hline \multirow[b]{3}{*}{ Nutrient intakes } & \multicolumn{2}{|c|}{ Non-smoker } & \multicolumn{3}{|c|}{ Current smoker } & \multirow{3}{*}{$\begin{array}{l}\text { Test for } \\
\text { linear trend } \\
P \text { value }\end{array}$} & \multirow[b]{3}{*}{$\begin{array}{c}\text { Residua } \\
\text { SD }^{L}\end{array}$} \\
\hline & \multirow{2}{*}{$\begin{array}{c}\begin{array}{c}\text { Never } \\
\text { smoked } \\
\text { cigarettes }\end{array} \\
\begin{array}{c}\text { Adj. mean } \\
(n=265-330)\end{array}\end{array}$} & \multirow{2}{*}{$\begin{array}{c}\begin{array}{c}\text { Previously } \\
\text { smoked } \\
\text { cigarettes }\end{array} \\
\begin{array}{c}\text { Adj. mean } \\
(n=474-575)\end{array} \\
\end{array}$} & \multirow{2}{*}{$\begin{array}{c}\begin{array}{c}\text { Cigar/ } \\
\text { pipe }\end{array} \\
\begin{array}{c}\text { Adj. mean } \\
(n=35-38)\end{array}\end{array}$} & \multirow{2}{*}{$\begin{array}{c}<20 \\
\begin{array}{c}<i g a r e t t e s \\
\text { day }^{-1}\end{array} \\
\begin{array}{c}\text { Adj. mean } \\
(n=98-119)\end{array} \\
\end{array}$} & \multirow{2}{*}{$\begin{array}{c}\geqslant 20 \\
\text { cigarettes }_{\text {day }^{-1}} \\
\begin{array}{c}\text { Adj. mean } \\
(n=35-45)\end{array}\end{array}$} & & \\
\hline & & & & & & & \\
\hline \multicolumn{8}{|l|}{ Antioxidant micronutrients } \\
\hline Plasma vitamin $\mathrm{C}\left(\mu \mathrm{mol} \mathrm{I}^{-1}\right)$ & 32.7 & 34.3 & 31.5 & 26.6 & 24.5 & 0.0002 & 19.3 \\
\hline Plasma $\beta$-carotene $\left(\mu \mathrm{mol} \mathrm{I}^{-1}\right)$ & 0.358 & 0.343 & 0.285 & 0.279 & 0.286 & 0.0006 & 0.23 \\
\hline Plasma $\alpha$-carotene $\left(\mu \mathrm{mol} \mathrm{I}^{-1}\right)^{\dagger}$ & 0.055 & 0.051 & 0.046 & 0.040 & 0.039 & $<0.0001$ & 0.26 \\
\hline Plasma lycopene $\left(\mu \mathrm{mol} \mathrm{I}^{-1}\right)^{\ddagger}$ & 0.195 & 0.191 & 0.230 & 0.135 & 0.149 & 0.0002 & 0.35 \\
\hline Plasma lutein $\left(\mu \mathrm{mol} \mathrm{I}^{-1}\right)^{\dagger}$ & 0.319 & 0.300 & 0.312 & 0.278 & 0.277 & 0.005 & 0.20 \\
\hline$\beta$-cryptoxanthin $\left(\mu \mathrm{mol} \mathrm{I}^{-1}\right)^{\dagger}$ & 0.100 & 0.090 & 0.077 & 0.066 & 0.067 & $<0.0001$ & 0.35 \\
\hline$\alpha$-tocopherol $\left(\mu \mathrm{mol} \mathrm{I}^{-1}\right)^{\dagger}$ & 33.0 & 32.4 & 31.6 & 31.6 & 30.9 & 0.1 & 0.12 \\
\hline$\alpha$-tocopherol/cholesterol $\left(\mathrm{mmol}^{-1} \mathrm{~mol}^{-1}\right)^{\dagger}$ & 6.12 & 6.05 & 5.83 & 6.03 & 5.73 & 0.2 & 0.10 \\
\hline$\gamma$-tocopherol $\left(\mu \mathrm{mol} \mathrm{I}^{-1}\right)^{\dagger}$ & 1.95 & 2.02 & 2.01 & 1.90 & 1.89 & 0.5 & 0.19 \\
\hline EGRAC (ratio) ${ }^{\S}$ & 1.28 & 1.28 & 1.29 & 1.34 & 1.33 & $<0.0001$ & 0.08 \\
\hline \multicolumn{8}{|l|}{ Other micronutrients } \\
\hline $\left.\operatorname{PLP}(\mathrm{nmol} \mathrm{I})^{-1}\right)^{\dagger}$ & 28.5 & 27.9 & 24.6 & 20.5 & 19.0 & $<0.0001$ & 0.23 \\
\hline Plasma retinol $\left(\mu \mathrm{mol} \mathrm{I}^{-1}\right)^{\dagger}$ & 1.99 & 1.98 & 1.90 & 1.86 & 1.81 & 0.006 & 0.12 \\
\hline Serum folate $\left(\mathrm{nmol} \mathrm{I}^{-1}\right)^{f^{\prime}}$ & 11.0 & 11.1 & 9.84 & 9.23 & 9.57 & 0.004 & 0.24 \\
\hline Red cell folate $\left(\mathrm{nmol} \mathrm{I}^{-1}\right)^{\dagger}$ & 401 & 445 & 368 & 346 & 361 & 0.001 & 0.21 \\
\hline ETKAC (ratio) $)^{\S}$ & 1.16 & 1.16 & 1.16 & 1.17 & 1.18 & 0.3 & 0.05 \\
\hline Serum vitamin $\mathrm{B}_{12}\left(\mathrm{pmol}^{-1}\right)^{\dagger}$ & 204 & 217 & 202 & 209 & 211 & 0.6 & 0.18 \\
\hline Plasma zinc $\left(\mu \mathrm{mol} \mathrm{I}^{-1}\right)$ & 13.6 & 13.3 & 13.5 & 13.9 & 13.6 & 0.3 & 2.16 \\
\hline Serum ferritin $\left(\mu \mathrm{g} \mathrm{I}^{-1}\right)^{\dagger}$ & 65.2 & 60.1 & 73.8 & 61.0 & 47.0 & 0.1 & 0.41 \\
\hline $25-\mathrm{OH}$-vitamin D $\left(\mathrm{nmol} \mathrm{I}^{-1}\right)^{\dagger}$ & 38.0 & 37.8 & 34.4 & 33.9 & 28.8 & 0.0003 & 0.20 \\
\hline Plasma calcium $\left(\mathrm{mmol} \mathrm{I}^{-1}\right)^{\dagger}$ & 2.32 & 2.32 & 2.31 & 2.30 & 2.34 & 0.7 & 0.03 \\
\hline Plasma copper $\left(\mu \mathrm{mol} \mathrm{I}^{-1}\right)^{\dagger}$ & 16.9 & 18.2 & 18.2 & 18.9 & 20.6 & $<0.0001$ & 0.08 \\
\hline \multicolumn{8}{|l|}{ Lipids } \\
\hline Cholesterol $\left(\mathrm{mmol} \mathrm{I}^{-1}\right)^{\|}$ & 5.46 & 5.55 & 5.59 & 5.38 & 5.54 & 0.8 & 1.32 \\
\hline HDL cholesterol $\left(\mathrm{mmol} \mathrm{I}^{-1}\right)^{\dagger, \|}$ & 1.13 & 1.11 & 1.30 & 1.06 & 1.12 & 0.3 & 0.15 \\
\hline Triglycerides $\left(\mathrm{mmol} \mathrm{I}^{-1}\right)^{\dagger}$ & 1.37 & 1.46 & 1.32 & 1.56 & 1.45 & 0.05 & 0.20 \\
\hline
\end{tabular}

${ }^{*}$ Adjusted for age category, sex, domicile and intake of corresponding nutrient (excludes supplement takers to prevent severe skewness of data). Excludes those without a full 4-day diet diary. Weighted means are presented to correct for non-proportional sampling (see subjects and methods section). Analysis of variance used to obtain means with nutrient status indices as the dependent variable, and smoking status as the independent variable. Smokers using both cigarettes and cigars/pipe are classed as cigarette smokers. Variables were transformed to obtain a normal distribution, and means back-transformed: ${ }^{\dagger}$ log, ${ }^{\ddagger}$ square root, ${ }^{\S}$ reciprocal.

"Adjusted for fat intake.

III Across the five smoking groups.

LOverall residual standard deviation on the transformed scale where relevant.

EGRAC, erythrocyte glutathione reductase activation coefficient (riboflavin status); PLP, plasma pyridoxal phosphate; ETKAC, erythrocyte transketolase activation coefficient (thiamin status); Adj., adjusted.

Not significant when $P>0.01$.

$\gamma$-tocopherol indices). Cigarette smoking was also inversely correlated with plasma pyridoxal phosphate (PLP), plasma retinol, serum folate, red cell folate (RCF) and $25-\mathrm{OH}$ vitamin $\mathrm{D}$, and directly correlated with plasma copper.

After further adjustment for sociodemographic, health and drug usage variables, significance fell outside the $1 \%$ level $(P>0.01)$ for plasma $\beta$-carotene $(P=0.03)$, lutein $(P=0.17)$, retinol $(P=0.02)$ and 25 $\mathrm{OH}$ vitamin $\mathrm{D}(P=0.03)$ (not presented).

Previous cigarette smokers, both those who had given up $>2$ years (group $\mathrm{A} ; n=456$ ) or $\leqslant 2$ years (group B; $n=28$ ) before, had higher mean values for plasma copper than those who had never smoked cigarettes, after adjustment for age, sex and domicile (not presented). This relationship remained significant (group A $P=0.0001$; group B $P=0.02$ ) after further adjustment for sociodemographic, health and drug usage variables. Red cell folate was higher in those who had given up cigarettes $>2$ years before than those who had never smoked cigarettes (both before $P=0.004$, and after $P=0.04$, further adjustment). No other significant differences between previous cigarette smokers or cigar/pipe smokers and never smokers with respect to mean micronutrient and lipid status were observed after adjustment for age, sex and domicile (not presented).

The relationship between smoking status and other factors associated with health is shown in Table 6. After adjustment for age, sex and domicile, cigarette smoking was directly correlated with $\alpha_{1}$-antichymotrypsin (ACT), alkaline phosphatase, neutrophils and white cells, and inversely correlated with body mass index, mid-upper arm circumference and grip strength. After 
Table 6 Relationship between smoking status and other health indicators*

\begin{tabular}{|c|c|c|c|c|c|c|c|}
\hline \multirow[b]{3}{*}{ Health indicators } & \multicolumn{2}{|c|}{ Non-smoker } & \multicolumn{3}{|c|}{ Current smoker } & \multirow{3}{*}{$\begin{array}{l}\text { Test for } \\
\text { linear trend } \\
P \text { value }\end{array}$} & \multirow[b]{3}{*}{$\begin{array}{l}\text { Residua } \\
\text { SD" }\end{array}$} \\
\hline & \multirow{2}{*}{$\begin{array}{c}\begin{array}{c}\text { Never } \\
\text { smoked } \\
\text { cigarettes }\end{array} \\
\begin{array}{c}\text { Adj. mean } \\
(n=274-346)\end{array}\end{array}$} & \multirow{2}{*}{$\begin{array}{c}\begin{array}{c}\text { Previously } \\
\text { smoked } \\
\text { cigarettes }\end{array} \\
\begin{array}{c}\text { Adj. mean } \\
(n=480-621)\end{array}\end{array}$} & \multirow{2}{*}{$\begin{array}{c}<20 \\
\begin{array}{c}\text { Cigar/ } \\
\text { pipe }\end{array} \\
\text { Adj. mean } \\
(n=28-39)\end{array}$} & \multirow{2}{*}{ 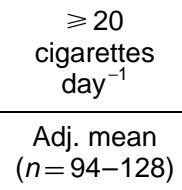 } & \multirow{2}{*}{$\begin{array}{c}\begin{array}{c}\text { cigarettes } \\
\text { day }^{-1}\end{array} \\
\begin{array}{c}\text { Adj. mean } \\
(n=30-47)\end{array}\end{array}$} & & \\
\hline & & & & & & & \\
\hline Systolic blood pressure $(\mathrm{mmHg})$ & 148 & 145 & 150 & 143 & 152 & 0.7 & 23.90 \\
\hline Diastolic blood pressure $(\mathrm{mmHg})$ & 76.7 & 75.1 & 75.8 & 72.1 & 76.5 & 0.03 & 13.98 \\
\hline$\alpha_{1}$-antichymotrypsin $\left(\mathrm{g} \mathrm{l}^{-1}\right)^{\dagger}$ & 0.385 & 0.394 & 0.373 & 0.436 & 0.439 & $<0.0001$ & 0.10 \\
\hline Alkaline phosphatase $\left(\mathrm{iu} \mathrm{I}^{-1}\right)^{\ddagger}$ & 88.2 & 84.9 & 89.0 & 93.4 & 107 & 0.0007 & 0.03 \\
\hline White cells count $\left(10^{9} I^{-1}\right)$ & 6.27 & 6.54 & 6.56 & 7.49 & 8.17 & $<0.0001$ & 0.11 \\
\hline$\gamma$-glutamyl transferase $\left(\mathrm{iu} \mathrm{L}^{-1}\right)^{\dagger}$ & 15.6 & 15.7 & 15.3 & 17.1 & 21.0 & 0.05 & 0.35 \\
\hline Neutrophils $\left(10^{9} \mathrm{I}^{-1}\right)^{\dagger}$ & 3.58 & 3.72 & 3.67 & 4.26 & 4.88 & $<0.0001$ & 0.16 \\
\hline Red cells $\left(10^{12} \mathrm{I}^{-1}\right)$ & 4.55 & 4.47 & 4.39 & 4.47 & 4.60 & 0.6 & 0.44 \\
\hline Haemoglobin $\left(\mathrm{g} \mathrm{dl}^{-1}\right)$ & 13.4 & 13.2 & 13.2 & 13.3 & 13.8 & 0.5 & 1.30 \\
\hline Albumin $\left(\mathrm{g} \mathrm{I}^{-1}\right)$ & 41.0 & 40.3 & 41.7 & 40.2 & 41.8 & 0.8 & 5.84 \\
\hline Homocysteine $\left(\mu \mathrm{mol} \mathrm{I}^{-1}\right)^{\dagger}$ & 15.9 & 16.3 & 15.6 & 17.1 & 16.6 & 0.2 & 0.16 \\
\hline Body mass index $\left(\mathrm{kg} \mathrm{m}^{-2}\right)$ & 25.7 & 25.9 & 25.2 & 23.5 & 23.9 & $<0.0001$ & 4.28 \\
\hline Mid-upper arm circumference $(\mathrm{cm})$ & 28.5 & 28.6 & 28.0 & 27.2 & 27.4 & $<0.0001$ & 3.49 \\
\hline Demi-span $(\mathrm{cm})$ & 75.0 & 75.4 & 75.0 & 74.7 & 74.9 & 0.4 & 3.98 \\
\hline Grip strength (kg) & 21.8 & 22.0 & 21.5 & 19.2 & 20.4 & 0.005 & 8.33 \\
\hline
\end{tabular}

*Adjusted for age category, sex and domicile. Excludes those without a full 4-day diary and those without a blood sample. Weighted means are presented to correct for non-proportional sampling (see subjects and methods section). Analysis of variance used with other health indicators as dependent variable and smoking status as independent variable. Smokers using both cigarettes and cigars/pipe are classed as cigarette smokers. Variables were transformed to obtain a normal distribution, and means back-transformed: ${ }^{\dagger}$ log, ${ }^{\ddagger}$ reciprocal.

§ Across the five smoking groups.

"Overall residual standard deviation on the transformed scale where relevant. Not significant when $P>0.01$.

Adj., adjusted.

further adjustment for sociodemographic variables significance fell outside the $1 \%$ level $(P>0.01)$ for alkaline phosphatase $(P=0.03)$ and grip strength $(P=0.07)$, and cigarette smoking, particularly light smoking, became inversely correlated with diastolic blood pressure $(P=0.003$; difference between means for light and never cigarette smokers $(P=0.008)$ (not presented).

After adjustment for age, sex and domicile, previous cigarette smokers who had given up smoking $\leqslant 2$ years before had significantly higher levels of ACT $(P=0.006)$, neutrophils $(P=0.02)$, white cells $(P=0.009)$ and homocysteine $(P=0.047)$ than those who had never smoked cigarettes (all of which lost significance $(P>0.05)$ after further adjustment for sociodemographic factors); and lower grip strength $(P=0.008)$, red cells $(P=0.002)$, haemoglobin $(P=0.047)$, and albumin $(P=0.01)$ than those who had never smoked cigarettes. Those who had given up $>2$ years before had lower haemoglobin $(P=0.03)$ than those who had never smoked cigarettes (not presented). After further adjustment for sociodemographic variables, previous smokers who had given up $>2$ years before had higher mean levels of ACT $(P=0.04)$ than those who had never smoked cigarettes, and those who had given up $\leqslant 2$ years before had higher MUAC $(P=0.03)$ than those who had never smoked cigarettes. No associations were observed between cigar/pipe smokers and factors in Table 6 after adjustment for age, sex and domicile (not presented).

The probability of current cigarette smokers reporting that they had bronchitis was greater than nonsmokers (6 vs. 3\%, $P=0.02$ ), and the chance of previous cigarette smokers having a self-reported long-standing illness was greater than those who had never smoked cigarettes (33 vs. $22 \%, P=0.005$ ), after adjustment for age, sex and domicile (not presented). After further adjustment for sociodemographic variables significance of these associations was $P=0.05$ and $P=0.02$, respectively. Smoking status was not associated with the probability of participants having other specific illnesses such as cancer, diabetes, stroke, heart attack, hypertension, stomach ulcer or other digestive complaints.

\section{Discussion}

Analysis in the present study was restricted to the 58\% of NDNS participants who provided a full 4-day diary, a blood sample and information on smoking. Although those participants not providing this information tended to differ demographically in terms of age, sex and region, there was no difference between cigarette smokers and non-smokers in those providing a food diary or a blood sample.

Smoking status in this study was assessed by questionnaire only. Biochemical markers such as 
serum cotinine were not available to determine the accuracy of the reported levels.

A possible source of bias in assessing nutrient intakes is the issue of under-reporting of energy intakes. Although results suggested some participants may have under-reported their energy intakes, the level of underreporting was similar across the five smoking groups, and thus results comparing smokers and non-smokers are likely to be unaffected by the under-reporting.

This study analysed trends across a logical progression of five smoking groups, from never smokers, through previous smokers, cigar/pipe smokers, light cigarette smokers to heavy cigarette smokers. Trends in nutrient intake data using a similar progression have been observed elsewhere ${ }^{15}$, and the numerous trends shown in this study support this approach.

The present study showed that cigarette smoking was inversely correlated with intakes of many antioxidant and other micronutrients, after adjustment for age, sex, domicile, total energy intake, sociodemographic, health and drug usage variables. Reduced intakes of micronutrients in cigarette smokers have also been reported by others $^{1-3}$. Previous cigarette smoking or cigar/pipe smoking was not associated with lower intakes of nutrients except for a lower intake of vitamin E observed in recent ex-smokers than those who had never smoked cigarettes. In accordance with this study other studies have found that cigarette smokers were less likely than non-smokers to consume fruit ${ }^{1,3}$, wholegrain and high fibre foods ${ }^{1}$ and more likely to consume whole milk ${ }^{1}$.

Cigarette smokers may therefore not only be at risk of chronic diseases due to the smoke-induced oxidant load but also due to low intakes of antioxidant nutrients ${ }^{1,3}$. Increasing the daily consumption of a variety of fruits and vegetables has recently been suggested as a good way of reducing the risk of chronic diseases ${ }^{24}$.

The UK Department of Health ${ }^{31}$ noted that to maintain body levels of vitamin $\mathrm{C}$ near to those of non-smokers, the vitamin $\mathrm{C}$ intake of smokers would need to be greater by up to $80 \mathrm{mg} \mathrm{day}^{-1}$ (the equivalent of one mediumsized orange or one average glass/carton of orange juice per day). The present study suggests that older people who smoke cigarettes need a higher vitamin $\mathrm{C}$ intake than non-smokers to obtain equivalent plasma levels, but the extra amount needed cannot be reported with confidence in this study due to the large variance surrounding the mean. Only about one-third of the difference in plasma vitamin $\mathrm{C}$ levels between smokers and non-smokers was explained by vitamin $C$ intakes in this study, and thus two-thirds may be largely attributable to the metabolic effects of smoking.

The susceptibility of micronutrients to cigarette smoke induced depletion has been investigated in in vitro studies, and varies widely ${ }^{12,16,32}$. Exposure of plasma to cigarette smoke has shown plasma vitamin C to be depleted quickly, while $\alpha$-tocopherol appears to be consumed only when ascorbic acid is almost completely destroyed ${ }^{16}$. Of the carotenoids in plasma, lycopene has been reported to be most susceptible, followed by lutein + zeaxanthin and $\beta$-carotene, with cryptoxanthin being less susceptible ${ }^{32}$. Retinol and $\gamma$ tocopherol appeared less susceptible to cigarette smoke degradation than other lipophilic micronutrients measured $^{32}$.

The present study showed that after adjustment for age, sex, domicile, nutrient intake, sociodemographic, health and drug usage variables, cigarette smoking was inversely correlated with a number of antioxidant micronutrient status indices including vitamin $\mathrm{C}$ and some carotenoids (but not vitamin E status indices) and with other micronutrient status indices, including plasma pyridoxal phosphate, red cell folate and serum folate.

Other studies have also reported significantly lower blood levels of vitamin $C^{4,5,33,34}$, some carotenoids such as $\alpha$-carotene ${ }^{33,34}, \beta$-carotene ${ }^{6,10,33,34}, \beta$-cryptoxanthin ${ }^{34}$, folate $^{9-11}$ and PLP ${ }^{35}$ in cigarette smokers. Like ours, other studies have also found no significant differences in vitamin $\mathrm{E}$ status indices ${ }^{5,34}$.

Since a number of factors including nutrient intakes have been corrected for in this analysis, it is likely that the observed differences between cigarette smokers and never smokers in nutrient status indices are largely due to the metabolic effects of cigarette smoking, and not the effects via food choices, sociodemographic factors or health status.

Cigarette smoking in this study was directly correlated with concentrations of the acute phase indicators: plasma copper, white cell count, ACT and neutrophils, after adjustment for age, sex, domicile and sociodemographic variables. Increased levels of acute phase indicators have also been reported by others and may reflect the induction by smoking of an inflammatory response which increases alveolar macrophages and neutrophils in the lungs ${ }^{21}$. The present study also found higher concentrations of some acute phase indicators in previous cigarette smokers compared to never cigarette smokers.

An inverse association was found between cigarette smoking, particularly light smoking and diastolic blood pressure, after further adjustment was made for sociodemographic factors. This result was unexpected but perhaps indicates that those cigarette smokers with high blood pressure have been medically advised to stop smoking.

Previous cigarette smoking was not associated with lower blood concentrations of micronutrients in this study, indicating that the adverse effects of smoking on micronutrient status may be reversible within the first 2 years of stopping smoking. It was not possible in this study to look at shorter periods of smoking cessation due to the small sample size of recent 
ex-smokers. A recent study has shown that cessation of smoking can improve plasma antioxidant status acutely (within $84 \mathrm{~h}$ ), despite no change in food habits ${ }^{14}$.

In conclusion, this study has found that older people who smoke cigarettes are at increased risk of suboptimal intakes and blood concentrations of antioxidants and other micronutrients, but the lower intakes found in cigarette smokers only partly explain their reduced blood indices. Cigar/pipe smoking and previous cigarette smoking were not generally associated with lower micronutrient intakes and status in this study, and this suggests that the adverse effects of cigarette smoking on nutrient intakes and status are reversible. Current and previous cigarette smoking was also associated with higher concentrations of acute phase indicators. Older people who smoke cigarettes should be encouraged to stop smoking, but where this is unsuccessful they need to have access to a range of palatable, micronutrient-rich foods and be encouraged to consume a healthier diet, and in particular, to consume a variety of fruits, fruit juice and vegetables.

\section{Acknowledgements}

The National Diet and Nutrition Survey of People aged 65 years or over was funded jointly by the Ministry of Agriculture, Fisheries and Food and the Department of Health and conducted by Social and Community Planning Research (SCPR) in conjunction with the Medical Research Council's Human Nutrition Research, the University of Newcastle, University College London and the University of Birmingham. The measurements of total plasma homocysteine were performed by $\mathrm{Dr}$ MA Mansoor, Central Hospital in Rogaland, Stavanger, Norway. Measurements of plasma pyridoxal phosphate were performed by Dr K Pentieva. CM Walmsley was supported by the Ministry of Agriculture, Fisheries and Food.

\section{References}

1 Subar AF, Harlan LC, Mattson ME. Food and nutrient intake differences between smokers and non-smokers in the US. AJPH 1990; 80: 1323-9.

2 McPhillips JR, Eaton CB, Gans KM et al. Dietary differences in smokers and nonsmokers from two southeastern New England communities. J. Am. Diet. Assoc. 1994; 94: 287-92.

3 Zondervan KT, Ocke MC, Smit HA, Seidell JC. Do dietary and supplementary intakes of antioxidants differ with smoking status? Int. J. Epidemiol. 1996; 25: 70-9.

4 Schectman G, Byrd JC, Gruchow HW. The influence of smoking on vitamin C status in adults. Am. J. Publ. Health 1989; 79: 158-62.

5 Faruque MDO, Khan MR, Rahman MDM, Ahmed F. Relationship between smoking and antioxidant nutrient status. Br. J. Nutr. 1995; 73: 625-32.

6 Margetts BM, Jackson AA. The determinants of plasma $\beta$ carotene: interaction between smoking and other lifestyle factors. Eur. J. Clin. Nutr. 1996; 50: 236-8.
7 Pamuk ER, Byers T, Coates RJ et al. Effect of smoking on serum nutrient concentrations in African-American women. Am. J. Clin. Nutr. 1994; 59: 891-5.

8 Marangon K, Herbeth B, Lecomte E et al. Diet, antioxidant status, and smoking habits in French men. Am.J. Clin. Nutr 1998; 67: 231-9.

9 Ortega RM, Lopez-Sobaler AM, Gonzalez-Gross MM et al. Influence of smoking on folate intake and blood folate concentrations in a group of elderly Spanish men. J. Am. Coll. Nutr. 1994; 13: 68-72.

10 Piyathilake CJ, Macaluso M, Hine RJ, Richards EW, Krumdieck CL. Local and systemic effects of cigarette smoking on folate and vitamin B12. Am. J. Clin. Nutr. 1994; 60: 559-66.

11 Mansoor MA, Kristensen O, Hervig T et al. Low concentrations of folate in serum and erythrocytes of smokers: methionine loading decreases folate concentrations in serum of smokers and nonsmokers. Clin. Chem. 1997; 43 2192-4.

12 Mezzetti A, Lapenna D, Pierdomenico SD et al. Vitamins E, C and lipid peroxidation in plasma and arterial tissue of smokers and non-smokers. Atherosclerosis 1995: 91-9.

13 Heimburger DC, Krumdieck CL, Alexander CB, Birch R, Dill SR, Bailey WC. Localised folic acid deficiency and bronchial metaplasia in smokers: hypothesis and preliminary report. Nutr. Int. 1987; 3: 54-60.

14 Brown AJ. Acute effects of smoking cessation on antioxidant status. Nutr. Biochem. 1996; 7: 29-39.

15 Bolton-Smith C, Woodward M, Brown CA, Tunstall-Pedoe $\mathrm{H}$. Nutrient intake by duration of ex-smoking in the Scottish Heart Health Study. Br. J. Nutr. 1993; 69: 315-32.

16 Eiserich JP, van der Vliet A, Handelman GJ, Halliwell B, Cross CE. Dietary antioxidants and cigarette smoke-indued biomolecular damage: a complex interaction. Am. J. Clin. Nutr. 1995; 62 (suppl.): 1490S-500S.

17 Heimburger DC, Alexander B, Birch R, Butterworth CE, Bailey WC, Krumdieck CL. Improvement in bronchial squamous metaplasia in smokers treated with folate and vitamin $\mathrm{B}_{12}$. Report of a preliminary randomized doubleblind intervention trial. J. Am. Med. Assoc. 1988; 259: $1525-$ 30.

18 Hoshino E, Shariff R, Van Gossum A et al. Vitamin E suppresses increased lipid peroxidation in cigarette smokers. J. Parent. Enteral Nutr. 1990; 14: 300-5.

19 Princen, HMG, van Poppel G, Vogelezang C, Buytenhek R, Kok FJ. Supplementation with vitamin $\mathrm{E}$ but not $\beta$-carotene in vivo protects low density lipoprotein from lipid peroxidation in vitro. Effects of cigarette smoking. Arterioscler. Thromb. 1992; 12: 554-62.

20 Alpha-Tocopherol, Beta Carotene Cancer Prevention Study Group. The effect of vitamin $\mathrm{E}$ and beta carotene on the incidence of lung cancer and other cancers in male smokers. New Engl. J. Med. 1994; 330: 1029-35.

21 Brown KM, Morrice PC, Duthie GG. Vitamin E supplementation suppresses indexes of lipid peroxidation and platelet counts in blood of smokers and nonsmokers but plasma lipoprotein concentrations remain unchanged. Am. J. Clin. Nutr. 1994; 60: 383-7.

22 van Poppel G, Hospers J, Buytenhek R, Princen HMG. No effect of $\beta$-carotene supplementation on plasma lipoproteins in healthy smokers. Am. J. Clin. Nutr. 1994; 60 730-4.

23 Cross CE, Traber MG. Cigarette smoking and antioxidant vitamins: the smoke screen continues to clear but has a way to go. Am.J. Clin. Nutr. 1997; 65: 562-3.

24 Hininger I, Chopra M, Thurnham DI et al. Effect of increased fruit and vegetable intake on the susceptibility of lipoprotein to oxidation in smokers. Eur. J. Clin. Nutr. 1997; 51: 601-6.

25 Rolls BJ. Ageing and appetite. Nutr. Rev. 1992; 50: 422-6.

26 Department of Health. The nutrition of elderly people. Report on Health and Social Subjects no. 43. London: HMSO, 1992. 
27 Heseker H, Schneider R. Requirement and supply of vitamin $\mathrm{C}, \mathrm{E}$ and $\beta$-carotene for elderly men and women. Eur.J. Clin. Nutr. 1994; 48: 118-27.

28 Finch S, Doyle W, Lowe C et al. National Diet and Nutrition Survey: People Aged 65 Years and Over. Vol. 1. Report of the Diet and Nutrition Survey. London: SO, 1998.

29 Hughes JM, Smithers G, Gay C et al. The British National Diet and Nutrition Survey of people aged 65 years or over: protocol and feasibility study. Proc. Nutr. Soc. 1995; 54: 631-43.

30 Goldberg GR, Black AE, Jebb SA et al. Critical evaluation of energy intake data using fundamental principles of energy physiology: 1 . Derivation of cut-off limits to identify underrecording. Eur. J. Clin. Nutr. 1991; 45: 569-81.

31 Department of Health. Dietary Reference Values for Food
Energy and Nutrient for the United Kingdom. Report on Health and Social Subjects no. 41. London: HMSO, 1991.

32 Handelman GJ, Packer L, Cross CE. Destruction of tocopherols, carotenoids, and retinol in human plasma by cigarette smoke. Am. J. Clin. Nutr. 1996; 63: 559-65.

33 Stegmayr B, Johansson I, Huhtasaari F, Moser U, Asplund K. Use of smokeless tobacco and cigarettes - effects on plasma levels of antioxidant vitamins. Int.J. Vit. Nutr. Res. 1993; 63: 195-200.

34 Ross MA, Crosely LK, Brown KM et al. Plasma concentrations of carotenoids and antioxidant vitamins in Scottish males: influences of smoking. Eur. J. Clin. Nutr. 1995; 49: 861-5.

35 Vermaak WJH, Ubbink JB, Barnard HC, Potgieter GM, van Jarsveld H, Groenewald AJ. Vitamin B6 nutrition status and cigarette smoking. Am. J. Clin. Nutr. 1990; 51: 1058-61. 